\title{
BI-LIPSCHITZ CONTACT INVARIANCE OF RANK
}

\author{
Nguyen Xuan Viet Nhan ${ }^{\mathrm{a}^{*}}$ \\ ${ }^{a}$ Basque Center for Applied Mathematics (BCAM), Alameda de Mazarredo, Bilbao, Bizkaia, Spain \\ ${ }^{*}$ Corresponding author: Email: nguyenxuanvietnhan@gmail.com
}

Article history

Received: April 29 ${ }^{\text {th }}, 2021$

Received in revised form: May $14^{\text {th }}, 2021 \mid$ Accepted: May $19^{\text {th }}, 2021$

Available online: January $27^{\text {th }}, 2022$

\begin{abstract}
We address the question of whether the Thom-Boardman symbol of a map germ is an invariant with respect to bi-Lipschitz right equivalence. We give a counterexample showing that in general the answer is negative. We prove that the rank of a map germ is a bi-Lipschitz contact invariant. Consequently, the first Thom-Boardman symbol and its length are bi-Lipschitz contact invariants.
\end{abstract}

Keywords: Bi-Lipschitz contact invariant; Rank; Thom-Boardman symbol.

DOI: http://dx.doi.org/10.37569/DalatUniversity.12.2.886(2022)

Article type: (peer-reviewed) Full-length research article

Copyright $@ 2022$ The author(s)

Licensing: This article is licensed under a CC BY-NC 4.0 


\section{INTRODUCTION}

In this paper, we focus on the problem of finding invariants with respect to biLipschitz contact equivalence. Some results related to this topic may be found, for example, in Birbrair and Mendes (2018) and Pham and Bui (2019). Roughly speaking, two map germs are called bi-Lipschitz contact equivalent if, after a bi-Lipschitz change of coordinates in the source, their graphs are bi-Lipschitz equivalent as sets. Bi-Lipschitz contact equivalence implies bi-Lipschitz equivalence between the zero sets of the germs, yet it is quite rigid in the sense that moduli do not appear. Namely, given a smooth family of polynomial maps, Ruas and Valette (2011) prove that there is a finite partition of the parameter space such that on each element of the partition, the family is bi-Lipschitz contact trivial. We would like to remark that such a partition does not always exist for the topological left-right equivalence. Thom (1962) gives a family of polynomial maps $f_{t}$ for which if $t_{1} \neq t_{2}, f_{t_{1}}$ and $f_{t_{2}}$ are not topologically left-right equivalent.

The Thom-Boardman symbol is a smooth contact invariant introduced by Boardman (1967) based on generalizing the idea of Thom on describing the orders of singularities. It plays a crucial role in classifying singularities of differentiable maps. We are interested in the question of whether the Thom-Boardman symbol is an invariant with respect to bi-Lipschitz equivalences. It is shown in Section 3 that in general the answer is negative even for bi-Lipschitz right equivalence.

In Section 4 we prove that the rank of a smooth map germ is a bi-Lipschitz contact invariant (Theorem 4.2). Our result is deduced from the nice property that if two germs are bi-Lipschitz left-right equivalent, then their first homogeneous parts are also bi-Lipschitz left-right equivalent (Lemma 4.1). Together with the order, the rank can be regarded as one of the most basic invariants for the bi-Lipschitz contact equivalence. A direct consequence of this result is that the first Thom-Boardman symbol and its length are bi-Lipschitz contact invariants (Corollary 4.3).

Throughout the paper, given $X \subset \mathbb{R}^{n}$ and two non-negative functions $f, g: X \rightarrow \mathbb{R}$, we write $f \lesssim g$ (or $g \gtrsim f$ ) if there is $C>0$ such that $f(x) \leq C g(x), \forall x \in X$, and write $f \sim g$ if $f \lesssim g$ and $g \lesssim f$.

\section{PRELIMINARIES}

\subsection{Bi-Lipschitz contact equivalence}

Two smooth germs $f, g:\left(\mathbb{R}^{n}, 0\right) \rightarrow\left(\mathbb{R}^{p}, 0\right)$ are called bi-Lipschitz left-right equivalent (or bi-Lipschitz $\mathscr{A}$-equivalent) if there are germs of bi-Lipschitz homeomorphisms $\varphi:\left(\mathbb{R}^{n}, 0\right) \rightarrow\left(\mathbb{R}^{n}, 0\right)$ and $\psi:\left(\mathbb{R}^{p}, 0\right) \rightarrow\left(\mathbb{R}^{p}, 0\right)$ such that $f \circ \varphi=\psi \circ g$. If $\psi$ coincides with the identity map, then $f$ and $g$ are called bi-Lipschitz right equivalent (or bi-Lipschitz $\mathscr{R}$-equivalent).

The germs $f$ and $g$ are said to be bi-Lipschitz contact equivalent (or bi-Lipschitz 
$K$-equivalent) if there are germs of bi-Lipschitz homeomorphisms $h:\left(\mathbb{R}^{n}, 0\right) \rightarrow\left(\mathbb{R}^{n}, 0\right)$ and $H:\left(\mathbb{R}^{n} \times \mathbb{R}^{p},(0,0)\right) \rightarrow\left(\mathbb{R}^{n} \times \mathbb{R}^{p},(0,0)\right)$ of the form $H(x, y)=(h(x), \theta(x, y))$ such that $H(x, f(x))=(h(x), g(h(x)))$ and $\theta(x, 0)=0$. If $h$ is the identity map then $f$ and $g$ are called bi-Lipschitz $C$-equivalent.

Given a smooth function germ $\xi:\left(\mathbb{R}^{n}, 0\right) \rightarrow(\mathbb{R}, 0)$, we write the Taylor expansion of $\xi$ at 0 as $T_{0} \xi=\sum_{k} \xi_{k}$ where $\xi_{k}$ is a homogeneous polynomial of degree $k$. The smallest $k$ such that $\xi_{k} \neq 0$ is called the order (or the multiplicity) of $\xi$ at 0 , denoted by $\operatorname{ord}(\xi, 0)$.

Let $f:\left(\mathbb{R}^{n}, 0\right) \rightarrow\left(\mathbb{R}^{p}, 0\right)$ be a smooth germ with components $f_{1}, \ldots f_{p}$. The rank of $f$ at 0 , denoted by $\operatorname{rank}(f, 0)$, is the rank of the Jacobian matrix $\left(\partial f_{i} / \partial x_{j}\right)$ at 0 . The order of $f$ at 0 is

$$
\operatorname{ord}(f, 0)=\min \left\{\operatorname{ord}\left(f_{i}, 0\right)\right\}_{i=1, \ldots, p} .
$$

The first homogeneous part of $f$ is the polynomial map $H_{f}=\left(f_{1, k}, \ldots, f_{p, k}\right)$ where $f_{i, k}$ is the homogeneous polynomial of degree $k=\operatorname{ord}(f, 0)$ in the Taylor expansion of $f_{i}$ at 0 .

Example 2.1. Consider $f(x, y)=\left(x^{2}+y^{3}, x^{2} y\right)$. It is obvious that $\operatorname{rank}(f, 0)=0, \operatorname{ord}(f, 0)=$ 2 and $H_{f}(x, y)=\left(x^{2}, 0\right)$.

We need the following lemmas for the next section. Results in these lemmas are well known and easy to prove. One may find an analogous statement of Lemma 2.2 for function germs in Birbrair et al. (2007), Theorem 2.4, and a different proof of Lemma 2.3 in Bivià-Ausina and Fukui (2017), Theorem 3. For convenience, the lemmas are provided here with simple proofs.

Lemma 2.2. Let $f, g:\left(\mathbb{R}^{n}, 0\right) \rightarrow\left(\mathbb{R}^{p}, 0\right)$ be smooth germs. If $f$ and $g$ are bi-Lipschitz $\mathscr{K}$-equivalent, then there is a germ of bi-Lipschitz homeomorphism $\varphi:\left(\mathbb{R}^{n}, 0\right) \rightarrow\left(\mathbb{R}^{n}, 0\right)$ and a constant $C \geq 1$ such that

$$
\frac{1}{C}\|f\| \leq\|g \circ \varphi\| \leq C\|f\|
$$

Proof.

By definition, there is a bi-Lipschitz homeomorphism $\varphi:\left(\mathbb{R}^{n}, 0\right) \rightarrow\left(\mathbb{R}^{n}, 0\right)$ such that $f$ and $\bar{g}=g \circ \varphi$ are bi-Lipschitz $C$-equivalent. It means that there is a germ of a biLipschitz homeomorphism $H:\left(\mathbb{R}^{n} \times \mathbb{R}^{p},(0,0)\right) \rightarrow\left(\mathbb{R}^{n} \times \mathbb{R}^{p},(0,0)\right)$ such that $H(x, f(x))=$ $(x, \bar{g}(x))$. Since $f(0)=\bar{g}(0)=0$, for any $x$ in a small neighborhood of 0 ,

$$
\begin{aligned}
\|f(x)\|=\|(x, f(x))-(x, f(0))\| & =\left\|H^{-1}(x, \bar{g}(x))-H^{-1}(x, \bar{g}(0))\right\| \\
& \leq C\|(x, \bar{g}(x))-(x, \bar{g}(0))\|=C\|\bar{g}(x)\|
\end{aligned}
$$

where $C$ is a Lipschitz constant of $H^{-1}$. Similarly, we also have $\|\bar{g}(x)\| \leq C\|f(x)\|$. The lemma is proved. 
Lemma 2.3. Let $f, g:\left(\mathbb{R}^{n}, 0\right) \rightarrow\left(\mathbb{R}^{p}, 0\right)$ be smooth germs. If $f$ and $g$ are bi-Lipschitz $\mathscr{K}$-equivalent, then $\operatorname{ord}(f, 0)=\operatorname{ord}(g, 0)$.

Proof.

The proof is similar to the proof of Lemma 4.2 in Nguyen et al., 2020. By Lemma 2.2 , there is a germ of bi-Lipschitz homeomorphism $\varphi:\left(\mathbb{R}^{n}, 0\right) \rightarrow\left(\mathbb{R}^{n}, 0\right)$ such that $\|f\| \sim$ $\|g \circ \varphi\|$. Write $\varphi=\left(\varphi_{1}, \ldots, \varphi_{n}\right)$. Note that $\varphi_{i}$ are Lipschitz function germs. Suppose that $\operatorname{ord}(f, 0)=r$ and ord $(g, 0)=s$. By a linear change of coordinates in the source, we may assume that $H_{f}(1,0, \ldots, 0) \neq 0$ and $H_{g}(1,0, \ldots, 0) \neq 0$ where $H_{f}$ and $H_{g}$ are the first homogeneous parts of $f$ and $g$ respectively. Restricting to the $x_{1}$-axis we have

$$
\left\|f\left(x_{1}, 0, \ldots, 0\right)\right\| \sim\left\|H_{f}\left(x_{1}, 0, \ldots, 0\right)\right\| \sim\left|x_{1}\right|^{r}
$$

and

$$
\left\|g\left(\varphi\left(x_{1}, 0, \ldots, 0\right)\right)\right\| \lesssim\left|x_{1}\right|^{s}(\text { since } \varphi \text { is Lipschitz })
$$

Thus,

$$
1 \sim \frac{\left\|g\left(\varphi\left(x_{1}, \ldots, 0\right)\right)\right\|}{\left\|f\left(x_{1}, 0, \ldots, 0\right)\right\|} \lesssim \frac{\left|x_{1}\right|^{s}}{\left|x_{1}\right|^{r}} .
$$

This implies that $r \leq s$. Exchanging $f$ with $g$, we obtain $s \leq r$. Therefore, $r=s$.

\subsection{Pseudo-tangent maps associated to bi-Lipschitz homeomorphisms}

Given a germ of a bi-Lipschitz homeomorphism $\varphi:\left(\mathbb{R}^{n}, 0\right) \rightarrow\left(\mathbb{R}^{n}, 0\right)$. Suppose that $\psi$ is the inverse of $\varphi$. For $m \in \mathbb{N}$, we define

$$
\varphi_{m}(x)=m \varphi\left(\frac{x}{m}\right), \quad \psi_{m}(x)=m \psi\left(\frac{x}{m}\right) .
$$

It is obvious that $\varphi_{m}$ and $\psi_{m}$ are bi-Lipschitz homeomorphisms of the same Lipschitz constants as those of $\varphi$ and $\psi$, respectively. By the Arzela-Ascoli theorem, there exists a subsequence $\left\{m_{i}\right\}$ such that $\varphi_{m_{i}}$ and $\psi_{m_{i}}$ converge uniformly to bi-Lipschitz maps $\varphi^{*}$ and $\psi^{*}$. In Sampaio (2016) shows that $\psi^{*}$ is the inverse of $\varphi^{*}$. We call such $\varphi^{*}$ a pseudo-tangent map associated to the bi-Lipschitz homeomorphism $\varphi$.

\section{THE THOM-BOARDMAN SYMBOL IS NOT A BI-LIPSCHITZ RIGHT INVARIANT}

\subsection{The Thom-Boardman symbol of a smooth map germ}

Our main reference for this section is Gibson's book (Gibson, 1979, Chapter 5).

Let $\mathscr{E}_{n}$ denote the algebra of all smooth function germs on $\mathbb{R}^{n}$ at 0 . Let $I$ be a finitely generated ideal in $\mathscr{E}_{n}$ and $f_{1}, \ldots, f_{p}$ be the generators of $I$ and $x_{1}, \ldots, x_{n}$ be a local system of coordinates at 0 in $\mathbb{R}^{n}$. For an integer $s \geq 1$, denote by $\Delta_{S} I$ the ideal $I+I_{s}$ where $I_{s}$ is the ideal generated by $s \times s$ minors of the Jacobian matrix $\left(\partial f_{i} / \partial x_{j}\right)$ (if $s>n$ put 
$I_{S}=0$ ). Note that the ideal $\Delta_{s} I$ does not depend on the choice of generators or the choice of coordinates. One has the inclusion of ideals

$$
I \subseteq \Delta_{n} I \subseteq \Delta_{n-1} I \subseteq \ldots \subseteq \Delta_{1} I
$$

Putting $\Delta^{s} I=\Delta_{n-s+1}$, we obtain

$$
I \subseteq \Delta^{1} I \subseteq \Delta^{2} I \subseteq \ldots \subseteq \Delta^{n} I
$$

The critical Jacobian extension of $I$ is the largest proper ideal $\Delta^{i_{1}} I$ in (1). Continuing the process, we obtain an ascending sequence of critical Jacobian extensions:

$$
I \subseteq \Delta^{i_{1}} I \subseteq \Delta^{i_{2}} \Delta^{i_{1}} I \subseteq \ldots \subseteq \Delta^{i_{k}} \Delta^{i_{k-1}} \ldots \Delta^{i_{1}} I=\mathfrak{m}
$$

where $\mathfrak{m}$ is the maximal ideal of $\mathscr{E}_{n}$. The non-increasing sequence $\left(i_{1}, i_{2}, \ldots\right)$ is called the Thom-Boardman symbol of the ideal $I$.

Let $f:\left(\mathbb{R}^{n}, 0\right) \rightarrow\left(\mathbb{R}^{p}, 0\right)$ be a smooth germ with components $f_{1}, \ldots, f_{p}$. The ThomBoardman symbol of $f$, denoted by $\mathscr{B}(f)$, is the Thom-Boardman symbol of the ideal generated by the components $f_{1}, \ldots, f_{p}$.

We can write

$$
\mathscr{B}(f)=(\underbrace{a_{1}, \ldots, a_{1}}_{\alpha_{1} \text { times }}, \underbrace{a_{2}, \ldots, a_{2}}_{\alpha_{2} \text { times }}, \ldots)
$$

where $a_{i}>a_{i+1} \geq 0$. We call $a_{i}$ the $i$-th Thom-Boardman symbol of $f$ and $\alpha_{i}$ the length of $a_{i}$. It follows from the definition that

$$
a_{1}=n-\operatorname{rank}(f, 0) \text {, and } \alpha_{1}=\operatorname{ord}(f, 0)-1 .
$$

Theorem 3.1 (Gibson, 1979, Theorem 5.2). The Thom-Boardman symbol is a smooth contact invariant, i.e., if two germs $f, g:\left(\mathbb{R}^{n}, 0\right) \rightarrow\left(\mathbb{R}^{p}, 0\right)$ are smooth $\mathscr{K}$-equivalent, then they have the same Thom-Boardman symbol.

It is natural to ask the following:

Question 3.2. Is the Thom-Boardman symbol a bi-Lipschitz right (resp. bi-Lipschitz leftright, bi-Lipschitz contact) invariant?

\subsection{A counterexample to Question 3.2}

Example 3.3. Consider the family $f_{t}(x, y)=x^{4}+t x^{2} y^{6}+y^{9}$. It is easy to check that $\mathscr{B}\left(f_{0}\right)=(2,2,2,1,1,1,1,1,0)$ and $\mathscr{B}\left(f_{t}\right)=(2,2,2,1,1,1,1,0)$ for $t \neq 0$. Moreover, this family is bi-Lipschitz right trivial by Nguyen et al. (2020), Theorem 7.9. Therefore, the Thom-Boardman symbol is not a bi-Lipschitz right invariant. 


\section{BI-LIPSCHITZ CONTACT INVARIANCE OF RANK}

Let $f, g:\left(\mathbb{R}^{n}, 0\right) \rightarrow\left(\mathbb{R}^{p}, 0\right)$ be smooth germs. We have the following results.

Lemma 4.1. If $f$ and $g$ are bi-Lipschitz, $\mathscr{A}$-equivalent, so are $H_{f}$ and $H_{g}$.

Proof.

Since $f$ and $g$ are bi-Lipschitz $\mathscr{A}$-equivalent, there are germs of bi-Lipschitz homeomorphisms $\varphi:\left(\mathbb{R}^{n}, 0\right) \rightarrow\left(\mathbb{R}^{n}, 0\right)$ and $\psi:\left(\mathbb{R}^{p}, 0\right) \rightarrow\left(\mathbb{R}^{p}, 0\right)$ such that $f \circ \varphi=\psi \circ g$.

By Lemma 2.3 we may assume ord $(f, 0)=\operatorname{ord}(g, 0)=k$. So we can write $f$ and $g$ as

$$
f(x)=H_{f}(x)+O\left(\|x\|^{k+1}\right) \text { and } g(x)=H_{g}(x)+O\left(\|x\|^{k+1}\right) .
$$

By the Arzela-Ascoli theorem, there exists a sequence $\left\{m_{i}\right\}$ of positive integers such that as $i$ tends to $\infty$, the sequence of maps $\left\{m_{i} \varphi\left(\frac{x}{m_{i}}\right)\right\}$ converges to a Lipschitz map germ $\varphi^{*}$, and the sequence of maps $m_{i}^{k} \psi\left(\frac{x}{m_{i}^{k}}\right)$ converges to a Lipschitz map $\psi^{*}$. We also know that $\psi^{*}$ is the inverse of $\varphi^{*}$. Since $H_{f}$ and $H_{g}$ are homogeneous polynomial maps of degree $k$, we have

$$
m_{i}^{k} H_{f}\left(\varphi\left(\frac{x}{m}\right)\right)=H_{f}\left(m_{i} \varphi\left(\frac{x}{m_{i}}\right)\right) \rightarrow H_{f}\left(\varphi^{*}(x)\right) \text { as } m_{i} \rightarrow \infty,
$$

and

$$
m_{i}^{k} \psi\left(H_{g}\left(\frac{x}{m_{i}}\right)\right)=m_{i}^{k} \psi\left(\frac{H_{g}(x)}{m_{i}^{k}}\right) \rightarrow \psi^{*}\left(H_{g}(x)\right) \text { as } m_{i} \rightarrow \infty .
$$

Therefore,

$$
\begin{aligned}
m_{i}^{k} f\left(\varphi\left(\frac{x}{m_{i}}\right)\right) & =m_{i}^{k}\left[H_{f}\left(\varphi\left(\frac{x}{m_{i}}\right)\right)+O\left(\left\|\frac{x}{m_{i}}\right\|^{k+1}\right)\right] \\
& =H_{f}\left(m_{i} \varphi\left(\frac{x}{m_{i}}\right)\right)+m_{i}^{k} O\left(\left\|\frac{x}{m_{i}}\right\|^{k+1}\right) \rightarrow H_{f}\left(\varphi^{*}(x)\right),
\end{aligned}
$$

and

$$
\begin{aligned}
\left\|m_{i}^{k} \psi\left(g\left(\frac{x}{m_{i}}\right)\right)-m_{i}^{k} \psi\left(\left(H_{g}\left(\frac{x}{m_{i}}\right)\right)\right)\right\| & \leq C m_{i}^{k}\left\|g\left(\frac{x}{m_{i}}\right)-H_{g}\left(\frac{x}{m_{i}}\right)\right\| \\
& =C m_{i}^{k} O\left(\left\|\frac{x}{m_{i}}\right\|^{k+1}\right) \rightarrow 0,
\end{aligned}
$$

where $C$ is a Lipschitz constant of $\psi$. Consequently,

$$
m_{i}^{k} \psi\left(g\left(\frac{x}{m_{i}}\right)\right) \rightarrow \psi^{*}\left(H_{g}(x)\right) \text { as } m_{i} \rightarrow \infty .
$$


Since

$$
f\left(\varphi\left(\frac{x}{m_{i}}\right)\right)=\psi\left(g\left(\frac{x}{m_{i}}\right)\right)
$$

so

$$
H_{f}\left(\varphi^{*}(x)\right)=\psi^{*}\left(H_{g}(x)\right) .
$$

The proof is completed.

Theorem 4.2. If $f$ and $g$ are bi-Lipschitz $\mathscr{K}$-equivalent, then $\operatorname{rank}(f, 0)=\operatorname{rank}(g, 0)$.

Proof.

Assume that $\operatorname{rank}(f, 0)=r$ and $\operatorname{rank}(g, 0)=s$. By smooth changes of coordinates of the source and the target we can assume that $f(x)=\left(x_{1}, \ldots, x_{r}, \tilde{f}_{r+1}(x), \ldots, \tilde{f}_{p}(x)\right)$ and $g(x)=\left(x_{1}, \ldots, x_{s}, \tilde{g}_{s+1}(x), \ldots, \tilde{g}_{p}(x)\right)$ where the orders of $\tilde{f}_{i}$ and $\tilde{g}_{j}$ are larger than 1.

By the hypothesis, there are germs of bi-Lipschitz homeomorphisms

$$
h:\left(\mathbb{R}^{n}, 0\right) \rightarrow\left(\mathbb{R}^{n}, 0\right) \text { and } H:\left(\mathbb{R}^{n} \times \mathbb{R}^{p},(0,0)\right) \rightarrow\left(\mathbb{R}^{n} \times \mathbb{R}^{p},(0,0)\right)
$$

with $H(x, y)=(h(x), \theta(x, y))$ such that $H(x, f(x))=(h(x), g(h(x)))$ and $\theta(x, 0)=0$.

Set $F(x)=(x, f(x))$ and $G(x)=(x, g(x))$. It is obvious that $H \circ F=G \circ h$. This means that $F$ and $G$ are bi-Lipschitz $\mathscr{A}$-equivalent. By Lemma 4.1, $H_{F}=\left(x, f^{\prime}\right)$ and $H_{G}=\left(x, g^{\prime}\right)$ are bi-Lipschitz $\mathscr{A}$-equivalent where $f^{\prime}(x)=\left(x_{1}, \ldots, x_{r}, 0, \ldots, 0\right)$ and $g^{\prime}(x)=$ $\left(x_{1}, \ldots, x_{s}, 0, \ldots, 0\right)$. More precisely, it is shown in the proof of Lemma 4.1 that there is a pseudo-tangent map $H^{*}=\left(h^{*}, \theta^{*}\right)$ associated to the map $H$ where $h^{*}$ is a pseudo-tangent map associated to $h$ such that $H^{*} \circ H_{F}=H_{G} \circ h^{*}$. Note that $\theta^{*}(x, y)=\lim _{m_{i} \rightarrow \infty} m_{i} \theta\left(\frac{x}{m_{i}}, \frac{y}{m_{i}}\right)$ where $\left\{m_{i}\right\}$ is a sequence of positive integers tending to infinity. Since $\theta(x, 0)=0$, $\theta^{*}(x, 0)=0$. This implies that $f^{\prime}$ and $g^{\prime}$ are bi-Lipschitz $\mathscr{K}$-equivalent. It follows that $\left\{f^{\prime}=0\right\}$ and $\left\{g^{\prime}=0\right\}$ are bi-Lipschitz equivalent as sets. Therefore, $r$ must be equal to $s$.

The corollary below follows from Lemma 2.3, Theorem 4.2 and (2).

Corollary 4.3. The first Thom-Boardman symbol and its length are bi-Lipschitz contact invariants.

\section{Remark 4.4.}

(1) All results in the paper are also true for complex analytic germs, as can be shown by following the same arguments.

(2) It is still unknown if the rank is a topological left-right invariant for complex analytic germs. 


\section{ACKNOWLEDGMENTS}

This paper is dedicated to Professors Hà Huy Vui and Ta Lê Loi on the special occasion of their birthdays. The author would like to thank the referee for carefully reading the manuscript and providing useful comments. The research was supported by the ERCEA 615655 NMST Consolidator Grant and also by the Basque Government through the BERC 2018-2021 program and by the Spanish Ministry of Science, Innovation and Universities: BCAM Severo Ochoa accreditation SEV-2017-0718.

\section{REFERENCES}

Birbrair, L., Costa, J. C. F., Fernandes, A., \& Ruas, M. A. S. (2007). K-bi-Lipschitz equivalence of real function-germs. Proceedings of the American Mathematical Society, 135(4), 1089-1095. https://doi.org/10.1090/S0002-9939-06-08566-2

Birbrair, L., \& Mendes, R. (2018). Lipschitz contact equivalence and real analytic functions. arXiv:1801.05842v1. https://doi.org/10.48550/arXiv.1801.05842

Bivià-Ausina, C., \& Fukui, T. (2017). Invariants for bi-Lipschitz equivalence of ideals. The Quarterly Journal of Mathematics, 68(3), 791-815. https://doi.org/10.1093/ qmath/hax002

Boardman, J. M. (1967). Singularities of differentiable maps. Publications Mathématiques de l'I.H.'E.S., 33, 21-57. https://doi.org/10.1007/BF02684585

Gibson, C. G. (1979). Singular points of smooth mappings (Research notes in mathematics, Vol. 25). Pitman.

Nguyen, N., Ruas, M. A. S., \& Trivedi, S. (2020). Classification of Lipschitz simple function germs. Proceedings of the London Mathematical Society, 121(1), 51-82. https://doi.org/10.1112/plms.12310

Pham, T. -S. \& Bui, N. T. N. (2019). Invariants of the bi-Lipschitz contact equivalence of continuous definable function germs. arXiv:1901.04479v1. https://doi.org/10.485 50/arXiv.1901.04479

Ruas, M. A. S., \& Valette, G. (2011). Co and bi-Lipschitz K-equivalence of mappings. $M$ -athematische Zeitschrift, 269(12), 293-308. https://doi.org/10.1007/s00209-010 $-0728-\mathrm{Z}$

Sampaio, J. E. (2016). Bi-Lipschitz homeomorphic subanalytic sets have bi-Lipschitz ho -meomorphic tangent cones. Selecta Mathematica: New Series, 22(2), 553-559. https://doi.org/10.1007/s00029-015-0195-9

Thom, R. (1962). La stabilité topologique des applications polynomiales (Topological stability of polynomial maps). L'Enseignement Mathématique, 8, 24-33. 